\title{
Runx3-Regulated Expression of Two Ntrk3 Transcript Variants in Dorsal Root Ganglion Neurons
}

\author{
Yuuki Ogihara, ${ }^{1,2}$ Tomoyuki Masuda, ${ }^{1,2}$ Shigeru Ozaki, ${ }^{2}$ Masaaki Yoshikawa, ${ }^{3}$ Takashi \\ Shiga $^{1,2}$
}

${ }^{1}$ Department of Neurobiology, Faculty of Medicine, University of Tsukuba, Ibaraki, Japan.

${ }^{2}$ Doctoral Program in Kansei, Behavioral and Brain Sciences, Graduate School of Comprehensive Human Sciences, University of Tsukuba, Ibaraki, Japan.

${ }^{3}$ Division of Anatomical Science, Department of Functional Morphology, Nihon University School of Medicine, Tokyo, Japan.

Y. Ogihara and T. Masuda contributed equally to this article.

Correspondence to: T. Masuda (tmasu@md.tsukuba.ac.jp).

Contract grant sponsor: Grants-in-Aid for Scientific Research of the MEXT, Japan; contract grant numbers: 15K08148, 24590256 and 26640024.

Running title: Runx3-Regulated Expression of Ntrk3s

Keywords: dorsal root ganglion; neurotrophic factor; transcription factor; neural network 


\begin{abstract}
Somatosensation is divided into proprioception and cutaneous sensation. Dorsal root ganglion (DRG) neurons project their fibers toward peripheral targets including muscles and skin, and centrally to the spinal cord. Proprioceptive DRG neurons transmit information from muscle spindles and Golgi tendon organs to the spinal cord. We previously showed that Runt-related transcription factor 3 (Runx3) is expressed in these neurons and their projections to the ventral spinal cord and muscle spindles are lost in Runx3-deficient (Runx $3^{-/}$) mouse embryos. Although Runx3 is likely to contribute to the fate decision and projection of proprioceptive DRG neurons, the precise roles for Runx3 in these phenomena are unknown. To identify genes regulated by Runx3 in embryonic DRGs, we performed microarray analyses using cDNAs isolated from wild-type and Runx $3^{-/}$DRGs of embryonic day (E) 12.5 and selected two transcript variants of the tyrosine kinase receptor $C(\operatorname{TrkC})$ gene. These variants, Ntrk3 variant 1 (Ntrk3-v1) and variant 2 (Ntrk3-v2), encode full-length and truncated receptors of neurotrophin-3, respectively. Using double in situ hybridization, we found that most of Ntrk3-v1 mRNA expression in E14.5 DRGs depended on Runx3 but that more than half of Ntrk3-v2 mRNA one were expressed in a Runx3-independent manner. Furthermore, our data revealed that the rate of Ntrk3-v1 and Ntrk3-v2 colocalization in DRGs changed from E14.5 to E18.5. Together, our data suggest that Runx3 may play a crucial role in the development of DRGs by regulating the expression of Ntrk3 variants and that DRG neurons expressing Ntrk3-v1 but not Ntrk3-v2 may differentiate into proprioceptive ones.
\end{abstract}




\section{INTRODUCTION}

Runt-related transcription factors (Runx) are characterized by their Runt domains. In mammals, they are subdivided into three subfamilies, that is, Runx1, 2, and 3 (Levanon and Groner, 2004; Inoue et al., 2008; Ito, 2008). Runx1 is involved in the differentiation of blood cells and is known as a causative gene of acute myeloid leukemia (Okuda et al., 1996; Ito, 2008; Ichikawa et al., 2013). Runx2 is essential for bone formation and acts as a causative gene for cleidocranial dysplasia (Komori et al., 1997; Otto et al., 1997; Ito, 2008). Runx3 is expressed in the gastrointestinal mucosa epithelium and has been shown to act as a tumor suppressor gene in the gastric cancer (Li et al., 2002; Ito, 2008). In addition, both Runx1 and 3 are expressed in the nervous system, especially in dorsal root ganglion (DRG) neurons at early stages of development. They play a pivotal role in the formation of subtypes and projections of DRG neurons (Inoue et al., 2002, 2008; Levanon et al., 2002; Chen et al., 2006a; Chen et al., 2006b; Kramer et al., 2006; Yoshikawa et al., 2007, 2013; Nakamura et al., 2008; Lallemend et al., 2012; Yang et al., 2013).

DRG neurons are responsible for the transmission of somatic information that is classified into nociception, mechanoreception, and proprioception. Nociceptive and mechanoreceptive DRG neurons project their fibers to the dorsal horn of the spinal cord and to the skin. Nociceptive DRG neurons express receptors for neurotrophic factors such as tyrosine kinase receptor A (TrkA) and Ret. Mechanoreceptive DRG neurons express various receptors for neurotrophic factors such as TrkB, TrkC, GDNF family receptor $\alpha 1-4$ (GFR $\alpha 1-4)$, and Ret (Inoue et al., 2002; Luo et al., 2009; Honma et al., 2010; Abdo et al., 2011; Lallemend and Ernfors, 2012; Yoshikawa et al., 2013). Conversely, proprioceptive DRG neurons express TrkC and project their fibers to the ventral horn and intermediate zone of the spinal cord, Golgi tendon organs and to muscle spindles, transmitting the information necessary for coordinated movement, such as tension of the muscle and the position of the limbs (Inoue et al., 2002; Lallemend and Ernfors, 2012). Previous studies showed that nonpeptidergic nociceptive DRG neurons express Runx1 and that proprioceptive DRG neurons express Runx3 (Levanon et al., 2002; Kramer et al., 2006; Yoshikawa et al., 2007; Inoue et al., 2008; Lallemend and Ernfors, 2012).

The role of Runx3 in DRG neuronal development has been analyzed by studying mouse embryos deficient for the Runx3 gene (Run $\times 3^{-/-}$). Several studies have revealed that Run $x 3^{-/-}$ embryos have deficiencies in the projections of their proprioceptive DRG neurons to both peripheral and central targets (Inoue et al., 2002; Levanon et al., 2002). More recently, by analyses using double knockout mouse embryos deficient for Bax and Runx3 genes, the defective projections of proprioceptive DRG neurons are not due to cell death, but due to 
changes in the fate of DRG neurons (Nakamura et al., 2008; Lallemend et al., 2012). Proprioceptive DRG neurons differentiate from precursor neurons coexpressing TrkC and TrkB, and Runx3 is involved in this differentiation (Kramer et al., 2006; Inoue et al., 2008). After embryonic day (E) 11.5, the precursor neurons coexpressing TrkC and TrkB are suppressed in their TrkB expression by Runx3 and they differentiate into TrkC-positive/TrkB-negative proprioceptive DRG neurons (Kramer et al., 2006; Inoue et al., 2007; Abdo et al., 2011). The fate of proprioceptive DRG neurons is likely to be converted into that of mechanoreceptive DRG neurons in Runx $3^{-/-}$mouse embryos and the projection of the proprioceptive fibers thus might be lost in these embryos. However, the expression of the TrkC protein is partly maintained in Run $x 3^{-/}$mouse DRGs (Nakamura et al., 2008). This observation led us to identify genes that contribute to the fate and projection of proprioceptive DRG neurons in the downstream of Runx3.

In this study, as a first step to elucidate the Runx3-regulated mechanism that controls the cell fate and projection of proprioceptive DRG neurons, we examined the expression pattern of downstream genes of Runx3 in DRG neurons. We found that Runx3 is involved in the development of DRG neurons by regulating the expression of two transcript variants of the TrkC gene.

\section{MATERIALS AND METHODS}

\section{Animals}

The generation and characterization of Runx3-deficient mice were performed as described previously (Inoue et al., 2002). Noon of the vaginal plug date was considered as embryonic day 0.5 (E0.5). After anesthetic treatment with isoflurane, E12.5-18.5 mouse embryos were excised. Genotyping was performed by polymerase chain reactions (PCRs) using primer pairs as described previously (Nakamura et al., 2008). All experiments using mice were performed according to the Guide for the Care and Use of Laboratory Animals of the University of Tsukuba.

\section{Microarray Analysis}

From E12.5 wild-type and Runx $3^{-/}$mouse embryos, DRGs (from cervical to lumbar levels) were dissected, and the samples were analyzed by use of an Agilent Expression Array (Agilent Technologies, California). $\log _{2}$ ratios representing the difference in the expression level were obtained on the basis of the expression level in wild-type mouse DRGs. 


\section{In Situ Hybridization}

In vitro transcription from the cDNA fragments of mouse Neurotrophic tyrosine kinase receptor type3 variant 1 (Ntrk3-v1, NM_008746, 1977-2638; 662 bp), mouse Neurotrophic tyrosine kinase receptor type3 variant 2 (Ntrk3-v2, NM_182809, 1686-2713; 1028 bp), and mouse Runx3 (NM_019732, 944-1938; 995 bp, Usui et al., 2012) was performed with a digoxigenin (DIG) or fluorescein (FLU) RNA labeling kit (Roche Diagnostics, Basel, Switzerland). Lumbar segments 1 and 2 of E14.5 wild-type or Run $\times 3^{-/-}$mouse embryos were dissected out and frozen in Tissue-Tek OCT compound (Sakura Finetech Japan, Tokyo, Japan). Transverse sections (10- $\mu \mathrm{m}$ thickness) were cut using a cryostat and collected onto silane-coated glass slides. (Agilent Technologies). After fixation with paraformaldehyde / $0.1 \mathrm{M}$ phosphate buffer for 10 min, the sections were dehydrated with $100 \%$ methanol for $1 \mathrm{~h}$ and acetylated with triethanolamine (Sigma-Aldrich, Missouri, USA) for 10 minutes. Hybridization was carried out for $18 \mathrm{~h}$ at $72^{\circ} \mathrm{C}$ with DIG-labeled cRNA probes $(500 \mathrm{ng} / \mathrm{ml})$. Prior to detection, sections were washed in $2 \times \mathrm{SSC}$ for $1 \mathrm{~h}$ at $72^{\circ} \mathrm{C}$ and for $30 \mathrm{~min}$ at room temperature. After incubation with normal goat serum (Vector Laboratories, Peterborough, UK) for $2 \mathrm{~h}$ at room temperature, hybridization was detected by treatment with sheep anti-DIG-AP, fab fragments (Roche Diagnostics, 1 : 10,000), sheep anti-FLU-AP, fab fragments (Roche Diagnostics; $1: 10,000$ ), sheep anti-DIG-POD, fab fragments (Roche Diagnostics; $1: 1000$ ), or sheep anti-FLU-POD, fab fragments (Roche Diagnostics; $1: 1000$ ) for 2 days at $4^{\circ} \mathrm{C}$. To detect alkaline phosphatase, we used the 2-hydroxy-3-naphthoic acid-2'-phenylanilide phosphate (HNPP) Fluorescent Detection Set (Roche Diagnostics). To detect peroxidase, we used the TSA Plus Cyanine 3 System (PerkinElmer, Massachusetts; 1:100) or TSA Plus Biotin Kit (PerkinElmer; 1:100) and Alexa Fluor 488-labeled streptavidin (Life Technologies, California; 1:1000). To calculate the ratio of positive cells, we obtained the total number of DRG neurons by Islet- $1 / 2$ staining described below and then calculated the percentage of various mRNA-expressing cells among the total number of DRG neurons per section. Data were statistically analyzed using Student's unpaired $t$-test.

\section{Immunohistochemistry}

After in situ hybridization, sections were incubated with a rat monoclonal antibody against Islet-1/2 (39.4D5, Developmental Studies Hybridoma Bank, Iowa; 1:200) overnight at $4^{\circ} \mathrm{C}$. Then, they were incubated with an Alexa Fluor 488-labeled goat anti-mouse IgG antibody (Life Technologies; 1:1000) at room temperature for $1 \mathrm{~h}$. The sections were sealed with VECTASHIELD Mounting Medium with 4',6-diamidino-2-phenylindole (DAPI, Vector Laboratories) and photographed using an AxioVision (Carl Zeiss, Jena, Germany). 


\section{Real-Time Quantitative Reverse Transcription PCR}

Total RNA was prepared from DRGs of E14.5 wild-type and Run $x 3^{-/-}$mouse embryos from thoracic to lumbar levels using an RNeasy Plus Micro Kit (Qiagen, Limburg, Netherlands). The cDNA was synthesized using a QuantiTect Reverse Transcription Kit (Qiagen). Using SYBR Premix Ex Taq II (Takara Bio, Shiga, Japan), real-time quantitative reverse transcription PCR (qRT-PCR) was carried out for 50 cycles by Thermal Cycler Dice Real Time System (TP800; Takara Bio). All of the reactions were conducted in duplicate for each cDNA sample and four animals from each genotype were used. The following PCR primers were used: Ntrk3-v1 forward, 5'-TGGTCTTTGAATACATGAAGCATGG-3', reverse, 5'-AGGCTATCTGACTGGCGATGTG-3' (amplicon size, 150 bp; Takara Bio); Ntrk3-v2, forward, 5'-GAACCTTTGGAACCCAGTGGAA-3', reverse, 5'-CACACATATTGACCAGAGACCAGGA-3' (amplicon size; 138 bp; Takara Bio); $18 S$ rRNA, forward, 5'-ACTCAACACGGGAAACCTCA-3', reverse, 5'-AACCAGACAAATCGCTCCAC-3' (Hokkaido System Science Co, Hokkaido, Japan). The I $8 S$ rRNA expression level was determined for each sample to normalize the data. Data were statistically analyzed by use of Student's unpaired $t$-test.

\section{RESULTS}

\section{Selection of Candidate Genes that Contribute to the Cell Fate Decision and Projection of} DRG Neurons

Microarray analyses were performed using DRGs from E12.5 wild-type $(n=15)$ and Runx $3^{-/}$( $n$ $=15$ ) mouse embryos. Based on previous studies on neural development, we obtained more than 20 genes that showed a difference in expression amount more than twice between wild-type and Runx $3^{-/}$mouse DRGs (Table 1). We then focused on two Ntrk3 genes encoding the $\mathrm{TrkC}$ receptor for neurotrophic factor neurotrophin-3 (NT3; Table 1). Neurotrophic tyrosine kinase receptor type3 variant 1 (Ntrk3-v1, NCBI Reference Sequence: NM_008746) and Neurotrophic tyrosine kinase receptor type3 variant 2 (Ntrk3-v2, NCBI Reference Sequence: NM_182809) are transcript variants of Ntrk3 genes. The results of these microarray analyses showed that the relative expression values ( $\log _{2}$ ratios) of Ntrk3-v1 in Runx $3^{-/-}$mouse DRGs were -2.71 and that those of Ntrk3-v2 were -1.26 compared with the wild-type values (Table 1). Previous experiments have revealed that NT3 acts as a chemoattractant for DRG neurites in vitro and is required for the innervation of TrkC-positive DRG fibers toward their targets in vivo (Genç et al., 2004; Usui et al., 2012). These results clearly suggest that the NT3/TrkC signaling pathway may be involved in the projection of proprioceptive DRG neurons. Although both of 
the cDNA sequences maintain a homology from extracellular to transmembrane domains, the sequences of their intracellular ones are different (Supporting Information Fig. 1; Menn et al., 1998). The Ntrk3-v1 gene has a tyrosine kinase domain, whereas the Ntrk3-v2 gene lacks this domain. Thus, Ntrk3-v1 and Ntrk-v2 proteins are considered to be a full-length TrkC with tyrosine kinase activity (full-length TrkC) and an incomplete TrkC (truncated TrkC), respectively (Ichinose and Snider, 2000; Cronk et al., 2002).

\section{Expression of Ntrk3 mRNAs Regulated by Runx3}

By performing in situ hybridization using specific cRNA probes for Ntrk3-v1 and Ntrk3-v2, we examined the Ntrk3-v1 and Ntrk3-v2 mRNA expression profiles in E14.5 wild-type and Runx $3^{-/-}$ DRGs (Fig. 1A-D). In these experiments, we used FLU-labeled cRNA probes to detect both Ntrk3-v1 and Ntrk3-v2 signals. Islet-1/2, a marker of pan-DRG neurons, was seen in the nucleus of DRG neurons, whereas Ntrk3-v1 and Ntrk3-v2 were expressed in the cytoplasm and the nucleus, respectively (Fig. 1A-D). We confirmed that several other cRNA probes for Ntrk3-v1 and Ntrk3-v2 (probes synthesized against other gene regions of Ntrk3-v1 and Ntrk3-v2) also showed the same expression patterns in the cytoplasm and the nucleus (data not shown). The percentage of Ntrk3-v1 and Ntrk3-v2 mRNA-positive DRG neurons among neurons expressing Islet-1/2 was calculated for both wild-type and Runx $3^{-/}$DRGs. We observed that 5.98 and $0.34 \%$ of DRG neurons expressed Ntrk3-v1 mRNA in E14.5 wild-type and Runx $3^{-/}$DRGs, respectively (Fig. 1A, B, E). Conversely, Ntrk3-v2-positive neurons were observed with $5.45 \%$ in E14.5 wild-type DRGs, and with $3.21 \%$ in Run $x 3^{-/}$ones (Fig. 1C-E). Therefore, the percentage rates of Ntrk3-v1- and Ntrk3-v2-positive neurons among total DRG neurons in Run $\times 3^{-/}$embryos as compared with those for wild-type ones were $5.7 \%$ and $58.8 \%$, respectively. Additionally, using qRT-PCR we performed rate-of-change analyses of the expression of Ntrk3-v1 and Ntrk3-v2 mRNAs in DRG neurons under Runx3 deficiency and confirmed that Ntrk3-v1 and Ntrk3-v2 expression levels in Runx $3^{-/}$DRGs showed a significant reduction compared with those in wild-type ones, although the degree of reduction in the level of Ntrk3-v2 mRNA was smaller than that in Ntrk3-v1 mRNA (Supporting Information Fig. 2A-C). Together, these results suggest that most of the Ntrk3-v1 mRNA expression in E14.5 DRGs depended on Runx3 and that more than half of the Ntrk3-v2 mRNA expression was independent of Runx3 in these neurons.

\section{Colocalization of Ntrk3-v1 and Ntrk3-v2 mRNAs in DRG Neurons}

Next, we examined the colocalization of Ntrk3-v1 and Ntrk3-v2 using a double in situ hybridization procedure. To detect Ntrk3-v1 and Ntrk3-v2 signals, we used FLU- and DIG-labeled cRNA probes, respectively. Ntrk3-v1 was expressed in the cytoplasm of DRG 
neurons, whereas Ntrk3-v2 and DAPI were seen in the nucleus (Fig. 2A-H). We observed that $63.1 \%$ of Ntrk3-v1 mRNA-positive DRG neurons coexpressed Ntrk3-v2 mRNA and that $67.6 \%$ of Ntrk3-v2 mRNA-positive DRG neurons coexpressed Ntrk3-v1 mRNA in E14.5 wild-type embryos (Fig. 2A-D, I, J). To further characterize Ntrk3-v2 and Ntrk3-v1 colocalization at later development, we examined the colocalization of Ntrk3-v1 and Ntrk3-v2 mRNA-positive DRG neurons in wild-type DRGs at E18.5 when proprioceptive DRG neurons have finished their projection to the ventral horn of the spinal cord. We observed that $89.1 \%$ of Ntrk3-v2 mRNA-positive DRG neurons coexpressed Ntrk3-v1 mRNA and 43.5\% of Ntrk3-v1 mRNA-positive DRG neurons coexpressed Ntrk3-v2 mRNA in E18.5 wild-type embryos (Fig. $2 \mathrm{E}-\mathrm{I}, \mathrm{K})$. These results showed that the colocalization rate of Ntrk3-v1 and Ntrk3-v2 mRNAs in DRG neurons had changed from E14.5 to E18.5.

\section{Colocalization of Ntrk3s mRNA and Runx3 mRNA in DRG Neurons}

Using the double in situ hybridization procedure, we also examined the colocalization of Ntrk3-v1 or Ntrk3-v2 with Runx3 in E14.5 wild-type DRGs. To detect Runx3 signals, we used the FLU-labeled cRNA probe; and for detection of Ntrk3-v1 and Ntrk3-v2 signals, DIG-labeled cRNA probes. We observed that $92.0 \%$ of Ntrk3-v1 mRNA-positive DRG neurons and $53.8 \%$ of Ntrk3-v2 mRNA-positive ones coexpressed Runx3 mRNA (Fig. 3A-D, I-L, Q, R). These results showed that the rate of colocalization of Ntrk3-v1 and Runx3 in E14.5 wild-type DRGs was nearly equal to the reduction rate of Ntrk3-v1-positive neurons in E14.5 Runx $3^{-/}$ones (Fig. 1E). These results further showed that the rate of colocalization of Ntrk3-v2 and Runx3 in wild-type DRGs was also equal to the reduction rate of Ntrk3-v2-positive neurons in Runx $3^{-/-}$mouse ones at E14.5 (Fig. 1E). Next, we examined the colocalization of Ntrk3-v1 or Ntrk3-v2 with Runx3 in E18.5 wild-type DRGs and found that $46.2 \%$ of Ntrk3-v1 mRNA-positive DRG neurons and 24.0\% of Ntrk3-v2 mRNA-positive DRG neurons coexpressed Runx3 (Fig. 3E-H, M-Q, S). These results showed that the colocalization rates of Ntrk3-v1 or Ntrk3-v2 with Runx3 in DRGs changed from E14.5 to E18.5. In other words, our results revealed that the rate of Ntrk3-v2 mRNA-positive and Runx3-negative DRG neurons increased at E18.5 and that Ntrk3-v1-positive and Runx3-negative DRG neurons emerged at the same stage.

\section{DISCUSSION}

In this study, as a first step to search for molecules that control the cell fate and projection of proprioceptive DRG neurons in the downstream of Runx3, we examined the expression of two transcript variants of the TrkC gene, Ntrk3-v1 and Ntrk3-v2. In mouse embryonic DRG neurons, 
we examined whether the expression of Ntrk3-v1 and Ntrk3-v2 depended on Runx3 or not. At E14.5, corresponding to the period when proprioceptive DRG fibers project to the intermediate zone, Ntrk3-v1 expression in Runx $3^{-/-}$DRGs was low, whereas more than half of the Ntrk3-v2 expression was observed in these DRGs in a Runx3-independent manner. Thus, these results suggest that the development of DRG neurons may be controlled through the Runx3-regulated expression of $\operatorname{Trk} C$ transcript variants.

\section{Projection of Proprioceptive DRG Neurons Regulated by Runx3}

Proprioceptive DRG neurons have been reported to use TrkC for responding to NT3-induced chemoattraction and extend their fibers to the muscle spindle and ventral horn of the spinal cord expressing NT3 (Fariñas et al., 1996; Chen et al., 2002). This axonal guidance mechanism is based on the observation that projections of proprioceptive DRG fibers to the ventral horn are lost in both $N T 3^{-/}$and Ntrk3 ${ }^{-/}$embryos (Ernfors et al., 1994; Patel et al., 2003; Genç et al., 2004; Usui et al., 2012). We previously examined the developmental patterns of all TrkC expression including Ntrk3-v1 and Ntrk3-v2 using anti-TrkC antibody (antibody against the extracellular domain of $\operatorname{TrkC}$ ). In Run $x 3^{-/-}$mouse embryos, in spite of the loss of proprioceptive DRG neurons, TrkC-expressing neurons are not completely lost, indicating that TrkC expression is partially maintained even in Runx $3^{-/}$mouse DRGs (Nakamura et al., 2008). From these results, it is quite difficult to explain the mechanism of the projection of proprioceptive DRG neurons by only the NT3/TrkC signaling pathway. The Ntrk3-v2 receptor without tyrosine kinase activity (truncated TrkC) is considered to be dominant-negative for the Ntrk3-v1 receptor with tyrosine kinase activity (full-length TrkC; Palko et al., 1999). Conversely, it is highly possible that the NT3/Ntrk3-v2 signaling has functions different from those of the NT3/Ntrk3-v1 signaling, because Ichinose and Snider (2000) showed that the total length of neurite outgrowth is maintained in Ntrk3-v2-transfected wild-type DRG neurons but that their outgrowth patterns are different from those of Ntrk3-v1-transfected ones. Furthermore, knockout mice for the entire extracellular domain of TrkC, which lack the functions of both Ntrk3-v1 and Ntrk3-v2, exhibit more serious abnormalities in the development of Merkel cells and projections of mechanoreceptive DRG neurons to Merkel cells than those in Ntrk3-v1-deficient mice lacking the tyrosine kinase domain of TrkC. These results suggest a different function of Ntrk3-v2 in the projection of mechanoreceptive DRG neurons (Cronk et al., 2002).

In the present results obtained by in situ hybridization, the expression of Ntrk3-v1 was almost lost, but that of Ntrk3-v2 was seen, in E14.5 Runx3 ${ }^{-/}$DRG neurons (Fig. 1E). Thus, at E14.5, corresponding to the stage of intensive axonal projection, the Ntrk3-v1 protein may be expressed in a Runx3-dependent manner in proprioceptive DRG neurons, but Ntrk3-v2, in a Runx3-independent manner in presumptive mechanoreceptive DRG neurons. Therefore, fibers 
of proprioceptive DRG neurons may be guided toward the ventral horn of the spinal cord via the NT3/Ntrk3-v1 signaling pathway under the control of Runx3. Knockdown experiments for Ntrk3-v1 in wild-type DRG neurons or rescue experiments for Ntrk3-v1 in Runx ${ }^{-/}$DRG neurons may further reveal the precise role for Ntrk3-v1 in the projection of proprioceptive DRG neurons.

\section{Transition of Colocalization of Ntrk3s and Runx3 in Embryonic DRG Neurons}

Our study showed that Ntrk3-v1 and Ntrk3-v2 exhibited different expression patterns in colocalization with Runx3. Whereas the majority of Ntrk3-v1 colocalized with Runx3 in E14.5 wild-type DRG neurons, about half of Ntrk3-v2 did not colocalize with Runx3 (Fig. 3Q, R). This result is consistent with the fact that more than half of the Ntrk3-v2-positive DRG neurons were present in E14.5 Runx $3^{-/}$mouse embryos (Fig. 1E). Furthermore, our study revealed that almost all Runx3- and Ntrk3-v2-double-positive DRG neurons coexpressed Ntrk3-v1 at E14.5 (Fig. 3R). Taken together, these results suggest that the subpopulation of Ntrk3-v2-positive DRG neurons lost in Runx $3^{-/}$embryos might be proprioceptive DRG neurons coexpressing Runx3 and Ntrk3-v1 and that another subpopulation, which was not lost in Runx $3^{-/-}$embryos, might be mechanoreceptive DRG neurons. In addition, the colocalization of Ntrk3-v1 or Ntrk3-v2 with Runx3 showed a different pattern between E14.5 and E18.5. In particular, about half of the Ntrk3-v1-positive DRG neurons did not express Runx3 at E18.5 (Fig. 3S). These results imply that the Ntrk3-v1 expression may be regulated by Runx3 in a stage-specific manner.

What type of neurons coexpress Ntrk3-v1 and Ntrk3-v2 in each stage? Furthermore, what function do Ntrk3-v1 and Ntrk3-v2 have in each stage? TrkB, a receptor for brain-derived neurotrophic receptor (BDNF), is present as two isoforms, full-length $\operatorname{TrkB}\left(\operatorname{TrkB} . \mathrm{tk}^{+}\right)$and truncated TrkB (TrkB.t1; Fenner, 2012). On the basis of these isoforms, three types of dimers, TrkB.tk ${ }^{+}$homodimers, TrkB.t1 homodimers, and TrkB.t1-TrkB.tk ${ }^{+}$heterodimers, are present in the brain (Fenner, 2012). TrkB.t1 homodimers are known to regulate the concentration of extracellular BDNF, change the morphology of neurons and glial cells, and activate the intracellular signaling (Fenner, 2012). Conversely, TrkB.t1-TrkB.tk ${ }^{+}$heterodimers have a dominant-negative function to inhibit the action of the TrkB.tk ${ }^{+}$signaling (Fenner, 2012). In the case of TrkC, it is not yet clear whether Ntrk3-v1 and Ntrk3-v2 form a heterodimer as in the case of TrkB, but it is highly possible that Ntrk3-v2 forms a heterodimer with Ntrk3-v1 and functions as a dominant-negative receptor. Because most of the Ntrk3-v1- and Ntrk3-v2-double-positive neurons coexpressed Runx3 at E14.5, they were supposedly proprioceptive DRG neurons. However, most of these double-positive neurons did not coexpress Runx3 at E18.5, suggesting that they might not be proprioceptive DRG neurons. At E18.5, DRG neurons have almost completed their projections to the targets and begun synapse 
formation (Mears and Frank, 1997; Ozaki and Snider, 1997). We cannot exclude the possibility that colocalization of Ntrk3-v1 and Ntrk3-v2 may be involved in the synapse formation of DRG neurons.

It is well known that hierarchical transcription factors are used to specify DRG sensory neurons. Although the expression of Ntrk3-v1 and Ntrk3-v2 may be controlled by Runx3, it is unclear whether Runx3 binds directly to the regulatory region of the Ntrk3 gene or acts through some other transcription factors. Further studies will clarify this issue.

\section{ACKNOWLEDGEMENTS}

The authors thank Dr. H. Takebayashi for the gift of plasmids (Runx3 cDNA), Dr. Y. Ito for providing Runx3-deficient animals. The authors also thank Dr. K. Senzaki for many helpful comments and technical assistance. 


\section{REFERENCES}

Abdo H, Li L, Lallemend F, Bachy I, Xu XJ, Rice FL, Ernfors P. 2011. Dependence on the transcription factor Shox 2 for specification of sensory neurons conveying discriminative touch. Eur J Neurosci 34:1529-1541.

Chen AI, de Nooij JC, Jessell TM. 2006a. Graded activity of transcription factor Runx3 specifies the laminar termination pattern of sensory axons in the developing spinal cord. Neuron 49:395-408.

Chen CL, Broom DC, Liu Y, de Nooij JC, Li Z, Cen C, Samad OA, Jessell TM, Woolf CJ, Ma Q. 2006b. Runx 1 determines nociceptive sensory neuron phenotype and is required for thermal and neuropathic pain. Neuron 49:365-377.

Chen HH, Tourtellotte WG, Frank E. 2002. Muscle spindle-derived neurotrophin 3 regulates synaptic connectivity between muscle sensory and motor neurons. J Neurosci 22:3512-3519.

Cronk KM, Wilkinson GA, Grimes R, Wheeler EF, Jhaveri S, Fundin BT, Silos-Santiago I, Tessarollo L, Reichardt LF, Rice FL. 2002. Diverse dependencies of developing Merkel innervation on the trkA and both full-length and truncated isoforms of trkC. Development 129: 3739-3750.

Ernfors P, Lee KF, Kucera J, Jaenisch R. 1994. Lack of neurotrophin-3 leads to deficiencies in the peripheral nervous system and loss of limb proprioceptive afferents. Cell 77:503-512.

Fariñas I, Yoshida CK, Backus C, Reichardt LF. 1996. Lack of neurotrophin-3 results in death of spinal sensory neurons and premature differentiation of their precursors. Neuron 17:10651078.

Fenner BM. 2012. Truncated trkB: Beyond a dominant negative receptor. Cytokine. Growth Factor Rev 23:15-24.

Genç B, Özdinler PH, Mendoza AE, Erzurumlu RS. 2004. A chemoattractant role for NT-3 in proprioceptive axon guidance. PLos Biol 2:e403.

Honma Y, Kawano M, Kohsaka S, Ogawa M. 2010. Axonal projections of mechanoreceptive dorsal root ganglion neurons depend on Ret. Development 137:2319-2328.

Ichikawa M, Yoshimi A, Nakagawa M, Nishimoto N, Watanabe-Okochi N, Kurokawa M. 2013. A role for RUNX1 in hematopoiesis and myeloid leukemia. Int J Hematol 97:726-734.

Ichinose T, Snider WD. 2000. Differential effects of TrkC isoforms on sensory axon outgrowth. J Neurosci Res 59:365-371.

Inoue K, Ozaki S, Shiga T, Ito K, Masuda T, Okado N, Iseda T, Kawaguchi S, Ogawa M, Bae SC, Yamashita N, Itohara S, Kudo N, Ito Y. 2002. Runx3 controls the axonal projection of proprioceptive dorsal root ganglion neurons. Nat Neurosci 5:946-954.

Inoue K, Ito K, Osato M, Lee B, Bae SC, Ito Y. 2007. The transcription factor Runx3 represses 
the neurotrophin receptor TrkB during lineage commitment of dorsal root ganglion neurons. J Biol Chem 282:24175-24184.

Inoue K, Shiga T, Ito Y. 2008. Runx transcription factors in neuronal development. Neural Dev $3: 20$.

Ito Y. 2008. RUNX genes in development and cancer: regulation of viral gene expression and the discovery of RUNX family genes. Adv Cancer Res 99:33-76.

Komori T, Yagi H, Nomura S, Yamaguchi A, Sasaki K, Deguchi K, Shimizu Y, Bronson RT, Gao YH, Inada M, Sato M, Okamoto R, Kitamura Y, Yoshiki S, Kishimoto T. 1997. Targeted disruption of $C b f a 1$ results in a complete lack of bone formation owing to maturational arrest of osteoblasts. Cell 89:755-764.

Kramer I, Sigrist M, de Nooij JC, Taniuchi I, Jessell TM, Arber S. 2006. A role for Runx transcription factor signaling in dorsal root ganglion sensory neuron diversification. Neuron 49:379-393.

Lallemend F, Ernfors P. 2012. Moleculer interactions underlying the specification of sensory neurons. Trends Neurosci 35:373-381.

Lallemend F, Sterzenbach U, Hadjab-Lallemend S, Aquino JB, Castelo-Branco G, Sinha I, Villaescusa JC, Levanon D, Wang Y, Franck MC, Kharchenko O, Adameyko I, Linnarsson S, Groner Y, Turner E, Ernfors P. 2012. Positional differences of axon growth rates between sensory neurons encorded by runx3. EMBO J 31:3718-3729.

Levanon D, Bettoun D, Harris-Cerruti C, Woolf E, Negreanu V, Eilam R, Bernstein Y, Goldenberg D, Xiao C, Fliegauf M, Kremer E, Otto F, Brenner O, Lev-Tov A, Groner Y. 2002. The Runx 3 transcription factor regulates development and survival of TrkC dorsal root ganglia neurons. EMBO J 21:3454-3463.

Levanon D, Groner Y. 2004. Structure and regulated expression of mammalian RUNX genes. Rev Oncogene 23:4211-4219.

Li QL, Ito K, Sakakura C, Fukamachi H, Inoue K, Chi XZ, Lee KY, Nomura S, Lee CW, Han SB, Kim HM, Kim WJ, Yamamoto H, Yamashita N, Yano T, Ikeda T, Itohara S, Inazawa J, Abe T, Hagiwara A, Yamagishi H, Ooe A, Kaneda A, Sugimura T, Ushijima T, Bae SC, Ito Y. 2002. Causal relationship between the loss of RUNX3 expression and gastric cancer. Cell 109:113-124.

Luo W, Enomoto H, Rice FL, Milbrandt J, Ginty DD. 2009. Moleculer identification of rapidly adapting mechanoreceptors and their developmental dependence on Ret signaling. Neuron 64:841-856.

Mears SC, Frank E. 1997. Formation of specific monosynaptic connections between muscle spindle afferents and motoneurons in the mouse. J Neurosci 17:3128-3135.

Menn B, Timsit S, Calothy G, Lamballe F. 1998. Differential expression of TrkC catalytic and 
noncatalytic isoforms suggests that they act independently or in association. J Comp Neurol 401:47-64.

Nakamura S, Senzaki K, Yoshikawa M, Nishimura M, Inoue K, Ito Y, Ozaki S, Shiga T. 2008. Dynamic regulation of the expression of neurotrophin receptors by Runx3. Development 135:1703-1711.

Okuda T, van Deursen J, Hiebert SW, Grosveld G, Downing JR. 1996. AML1, the target of multiple chromosomal translocations in human leukemia, is essential for normal fetal liver hematopoiesis. Cell 84:321-330.

Otto F, Thornell AP, Crompton T, Denzel A, Gilmour KC, Rosewell IR, Stamp GWH, Beddington RSP, Mundlos S, Olsen BR, Selby PB, Owen MJ. 1997. Cbfal, a candidate gene for cleidocranial dysplasia syndrome, is essential for osteoblast differentiation and bone development. Cell 89:765-771.

Ozaki S, Snider WD. 1997. Initial trajectories of sensory axons toward laminar targets in the developing mouse spinal cord. J Comp Neurol 380:215-229.

Palko ME, Coppola V, Tessarollo L. 1999. Evidence for a role of truncated trkC receptor isoforms in mouse development. J Neurosci 19:775-782.

Patel TD, Kramer I, Kucera J, Niederkofler V, Jessell TM, Arber S, Snider WD. 2003. Peripheral NT3 signaling is required for ETS protein expression and central patterning of proprioceptive sensory afferents. Neuron 38:403-416.

Usui N, Watanabe K, Ono K, Tomita K, Tamamaki N, Ikenaka K, Takebayashi H. 2012. Role of motoneuron-derived neurotrophin 3 in survival and axonal projection of sensory neurons during neural circuit formation. Development 139:1125-1132.

Yang FC, Tan T, Huang T, Christianson J, Samad OA, Liu Y, Roberson D, Davis BM, Ma Q. 2013. Genetic control of the segregation of pain-related sensory neurons innervating the cutaneous versus deep tissues. Cell Rep 5:1353-1364.

Yoshikawa M, Senzaki K, Yokomizo T, Takahashi S, Ozaki S, Shiga T. 2007. Runx1 selectively regulates cell fate specification and axonal projections of dorsal root ganglion neurons. Dev Biol 303:663-674.

Yoshikawa M, Murakami Y, Senzaki K, Masuda T, Ozaki S, Ito Y, Shiga T. 2013. Co-expression of Runx1 and Runx3 in mechanoreceptive dorsal root ganglion neurons. Dev Neurobiol 73:469-479. 


\section{Figure legends}

\section{Figure 1}

Rates of Ntrk3-v1 and Ntrk3-v2 mRNA-positive cells in Runx $3^{-/}$and wild-type DRG neurons. (A, B) Fluorescent double-stained images of Ntrk3-v1 mRNA (red) and Islet-1/2 protein (green) at E14.5 for wild-type (A) and Runx $3^{-/}$(B) DRGs of lumbar segments 1 and 2 (L1-2). Horizontal cross-sectioned slices of the DRG are shown. Insets show enlarged views of the areas surrounded by the white square. (C, D) Fluorescent double-stained images of Ntrk3-v2 mRNA (red) and Islet-1/2 protein (green) at E14.5 wild-type (A) and Runx $3^{-/}$(B) L1-2 DRGs. Horizontal cross-sectioned slices of the DRG are shown. Insets show enlarged views of the areas surrounded by the white square. (E) Quantification of Ntrk3-v1 and Ntrk3-v2 mRNA-positive DRG neurons in E14.5 wild-type (black bars; 15 sections for counting Ntrk3-v1-positive neurons, 20 sections for counting Ntrk3-v2-positive neurons) and Runx $3^{-/-}$ mouse embryos (white bars; 15 sections for counting Ntrk3-v1-positive neurons, 15 sections for counting Ntrk3-v2-positive neurons). The bars represent the means + SEM. ${ }^{*} p<0.01,{ }^{*} p<<$ 0.0001 . Scale bars: $50 \mu \mathrm{m}$.

\section{Figure 2}

Colocalization of Ntrk3-v1 and Ntrk3-v2 mRNAs in DRG neurons. (A-D) Triple-stained images of Ntrk3-v1 mRNA (A; red), Ntrk3-v2 mRNA (B; green), and DAPI (C; blue) in DRGs of lumbar segments 1 and 2 of E14.5 wild-type mouse embryos. Horizontal cross-sectioned slices of the DRG are shown. Dashed lines indicate the outer edge of the DRG, and insets show enlarged views of the areas surrounded by the white square. Scale bar: $50 \mu \mathrm{m}$. (E-H) Same images at E18.5. Scale bar: $50 \mu \mathrm{m}$. (I) Graph showing the ratio of the number of Ntrk3-v1 and Ntrk3-v2 mRNA coexpressing cells compared with that of Ntrk3-v1 or Ntrk3-v2 mRNA-positive cells in E14.5 (black bars; 14 sections) and E18.5 (white bars; 8 sections) wild-type DRGs. The bars represent the means + SEM. (J) Diagram showing the coexpression pattern of Ntrk3-v1 and Ntrk3-v2 mRNAs in E14.5 wild-type DRGs. (K) Same diagram for E18.5 wild-type DRGs.

\section{Figure 3}

Colocalization of Ntrk3-v1 or Ntrk3-v2 mRNA with Runx3 mRNA in DRG neurons. (A-D) Triple-stained images of Runx3 mRNA (A; red), Ntrk3-v1 mRNA (B; green), and DAPI (C; blue) in DRGs of lumbar segments 1 and 2 (L1-2) of E14.5 wild-type embryos. Horizontal cross-sectioned slices of the DRG are shown. Dashed lines indicate the outer edge of the DRG, and the inset shows an enlarged view of the area surrounded by the white square. Scale bar: 50 $\mu \mathrm{m}$. (E-H) Same images for E18.5 wild-type embryos. Scale bar: $100 \mu \mathrm{m}$. (I-L) Triple-stained 
images of Runx3 mRNA (I; red), Ntrk3-v2 mRNA (J; green), and DAPI (K; blue) in L1-2 DRGs of E14.5 wild-type embryos. Horizontal cross-sectioned slices of the DRG are shown. Dashed lines indicate the outer edge of the DRG, and insets show enlarged views of the areas surrounded by the white square. Scale bar: $50 \mu \mathrm{m}$. (M-P) Same images for E18.5 wild-type embryos. Scale bar: $100 \mu \mathrm{m}$. (Q) Graph showing the ratio of the number of Ntrk3-v1 or Ntrk3-v2 mRNA-positive cells coexpressing Runx3 compared with that of Ntrk3-v1 or Ntrk3-v2 mRNA-positive cells in E14.5 (black bars; 15 sections for counting Runx3- and/or Ntrk3-v1-positive neurons, 14 sections for counting Runx3- and/or Ntrk3-v2-positive neurons) and E18.5 (white bars; 9 sections for counting Runx3-and/or Ntrk3-v1-positive neurons, 10 sections for counting Runx3- and/or Ntrk3-v2-positive neurons) wild-type DRGs. The bars represent the means + SEM. (R) Diagram showing the coexpression pattern of Ntrk3-v1, Ntrk3-v2, and Runx3 mRNAs in E14.5 wild-type DRGs. (S) Same diagram for E18.5 wild-type DRGs.

\section{Supporting Information Figure 1}

Schematic diagram of Ntrk3-v1 and Ntrk3-v2 transcripts. Extracellular domain (EC), transmembrane domain (TM), tyrosine kinase domain (TK), non-catalytic 2 domain (NC2).

\section{Supporting Information Figure 2}

Regulation of the expression of Ntrk3-v1 and Ntrk3-v2 mRNAs by Runx3. (A) Amplification plots of qRT-PCR for Ntrk3-v1 mRNA levels in E14.5 wild-type (orange curves) and Runx $3^{-/-}$ (blue curves) DRGs. (B) Amplification plots of qRT-PCR for Ntrk3-v2 mRNA levels in E14.5 wild-type (red curves) and Runx $3^{-/}$(blue curves) DRGs. (C) The expression levels of Ntrk3-v1 and Ntrk3-v2 mRNAs were compared between the wild-type (black bars, $n=2$ ) and Runx $3^{-/-}$ (white bars, $n=3$ ) DRGs at E14.5, as determined by qRT-PCR. The bars represent the means + SEM. $* p<0.05, * * p<0.01$. 
Table 1. Partial list of genes with altered expression in Runx3-deficient DRGs

\begin{tabular}{llll}
\hline Entrez Gene ID & Symbol & Gene name & Log $_{2}$ expression ratio \\
\hline 15375 & Foxal & forkhead box A1 & -5.59 \\
15432 & Hoxd12 & homeobox D12 & -5.07 \\
16872 & Lhx4 & LIM homeobox protein 4 & -5.05 \\
16876 & Lhx9 & LIM homeobox protein 9 & -4.89 \\
13172 & Dbxl & developing brain homeobox 1 & -4.57 \\
14028 & Evx1 & even skipped homeotic gene 1 homolog & -4.54 \\
11925 & Neurog3 & neurogenin 3 & -4.51 \\
13166 & $*$ Dbh & dopamine beta hydroxylase & -4.39 \\
15376 & Foxa2 & forkhead box A2 & -3.69 \\
16871 & Lhx3 & LIM homeobox protein 3 & -3.67 \\
140477 & Dmbx1 & diencephalon/mesencephalon homeobox 1, transcript variant 1 & -3.09 \\
11921 & Atoh1 & atonal homolog 1 & -3.05 \\
18213 & $*$ Ntrk3 & neurotrophic tyrosine kinase, receptor, type 3, transcript variant 1 & -2.71 \\
16876 & Lhx9 & LIM homeobox protein 9, transcript variant 1 & -2.62 \\
94222 & Olig3 & oligodendrocyte transcription factor 3 & -2.52 \\
13390 & Dlx1 & distal-less homeobox 1 & -2.39 \\
320747 & $*$ Lingo4 & leucine rich repeat and Ig domain containing 4 & -2.29 \\
240725 & $*$ Sulf1 & sulfatase 1 & -2.25 \\
14813 & $*$ Grin2c & glutamate receptor, ionotropic & -2.16 \\
18096 & Nkx6-1 & NK6 homeobox 1 & -2.15 \\
15407 & Hoxb1 & homeobox B1 & -2.12 \\
19649 & $*$ Robo3 & roundabout homolog 3 & -2.05 \\
20231 & Nkx1-2 & NK1 transcription factor related, locus 2 & -2.00 \\
18213 & $* N t r k 3$ & neurotrophic tyrosine kinase, receptor, type 3, transcript variant 2 & -1.26 \\
12550 & $*$ Cdh1 & cadherin 1 & 2.17 \\
15566 & $*$ Htr7 & 5-hydroxytryptamine (serotonin) receptor 7 & 2.38 \\
\hline
\end{tabular}

*These genes do not encode transcription factors. 


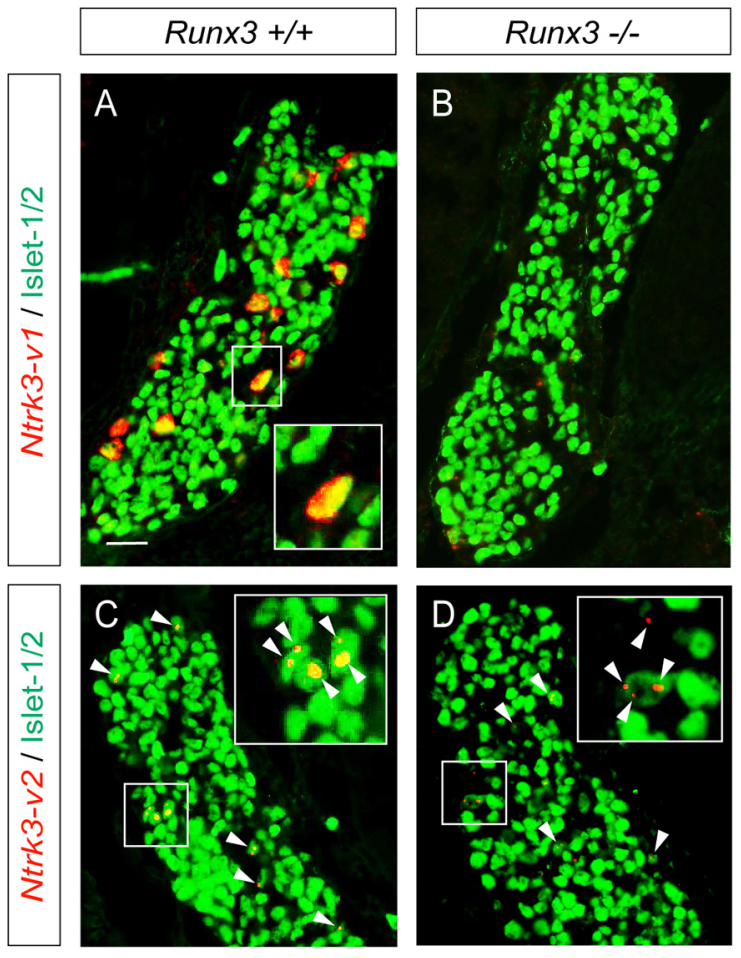

E

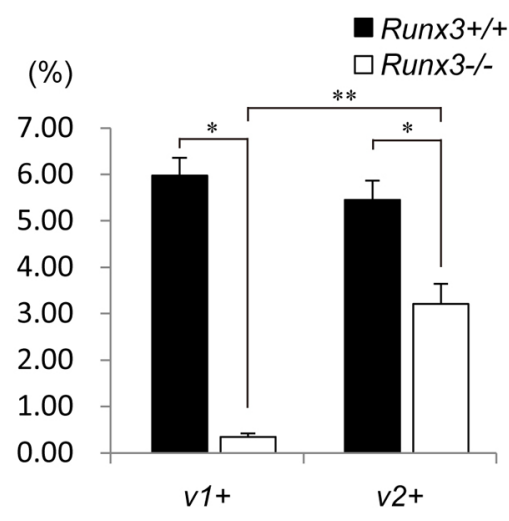

Fig. 1 


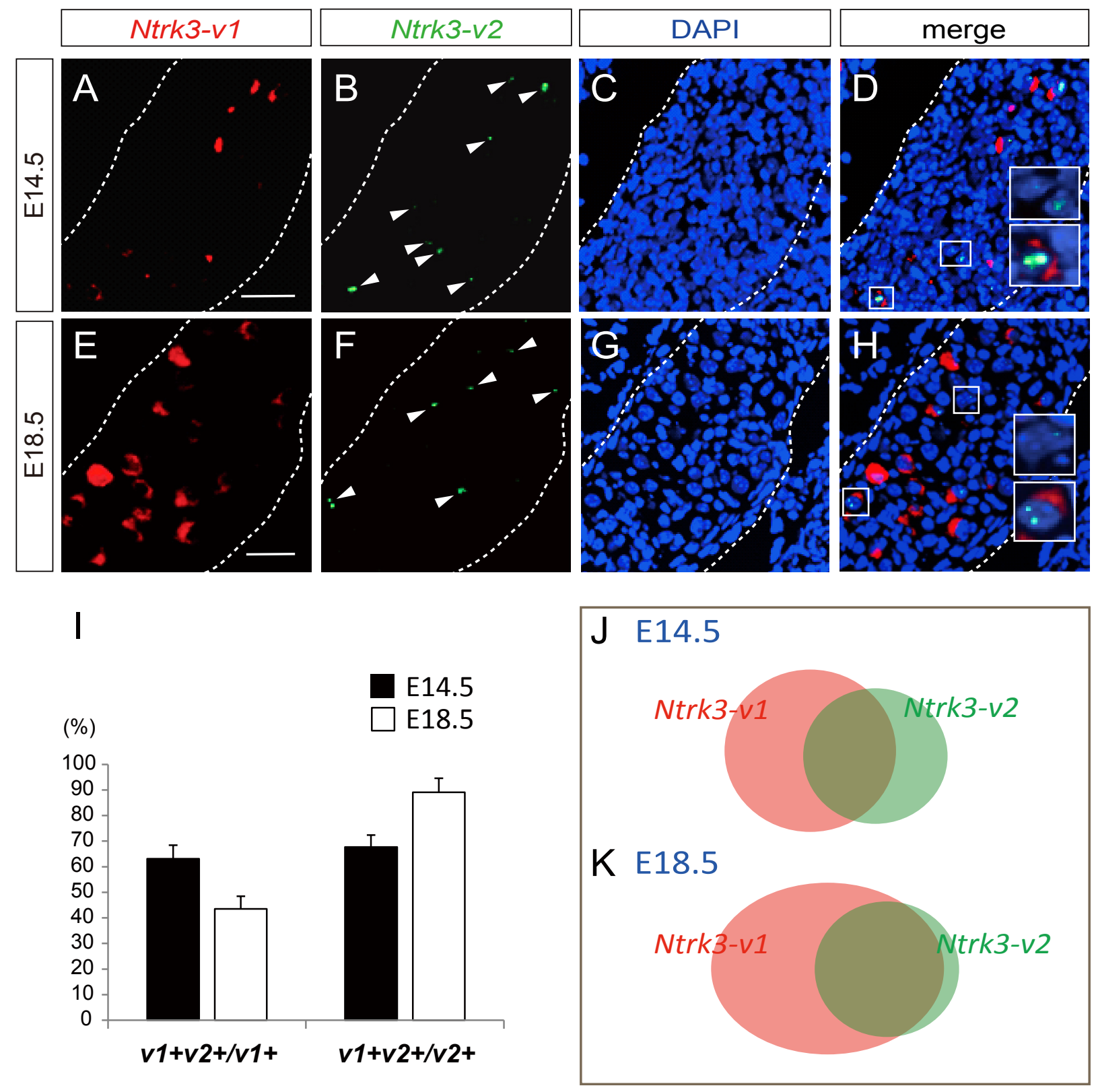

Fig. 2 

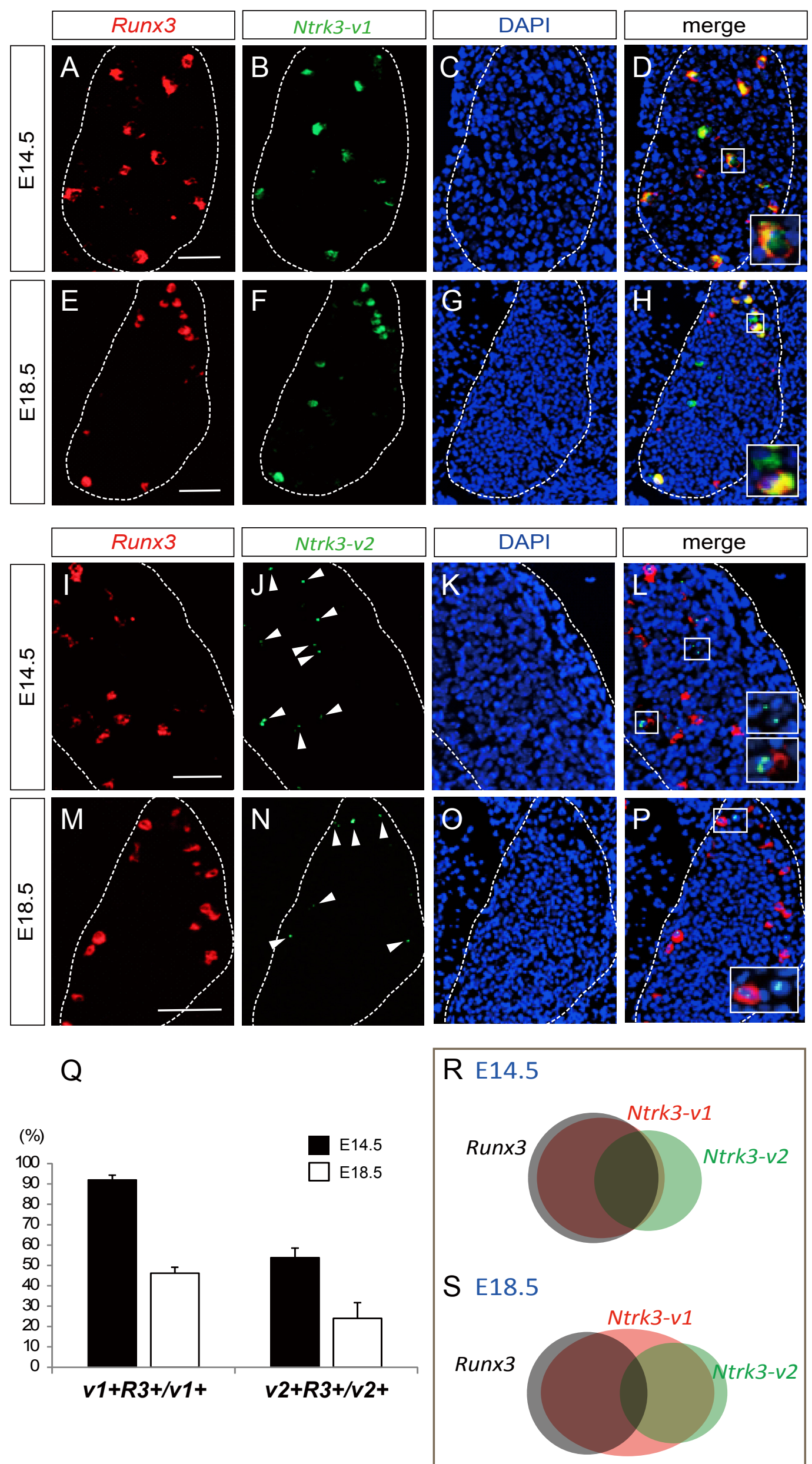

Fig. 3 
Ntrk3-v1 (RefSeq: NM_008746)

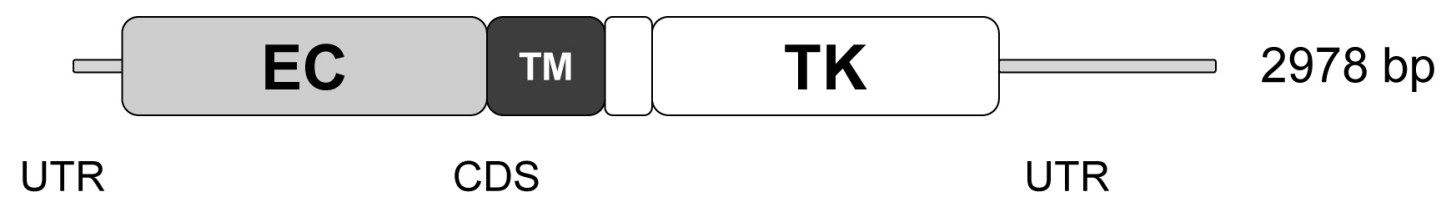

Ntrk3-v2 (RefSeq: NM_182809)

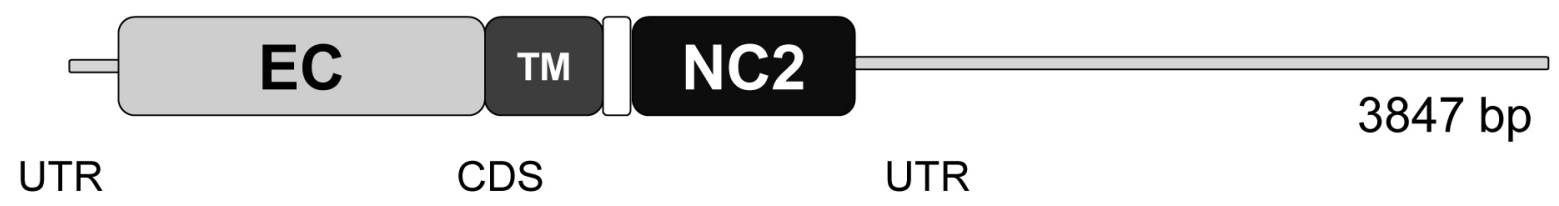

Supporting Information Fig. 1 
A

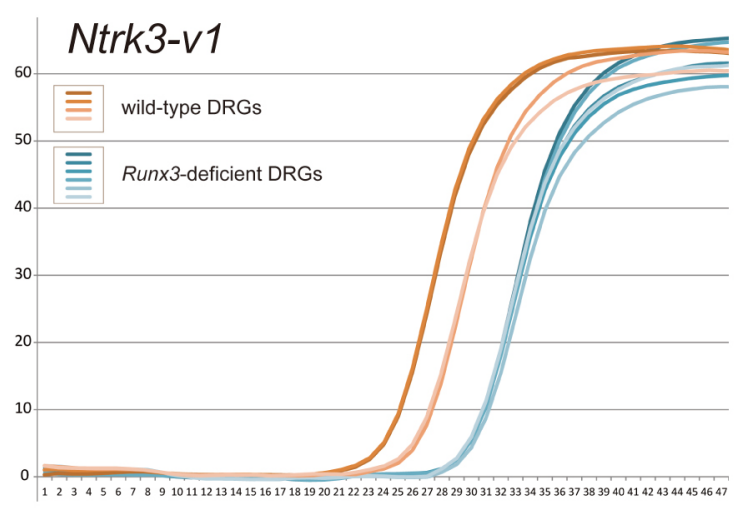

B

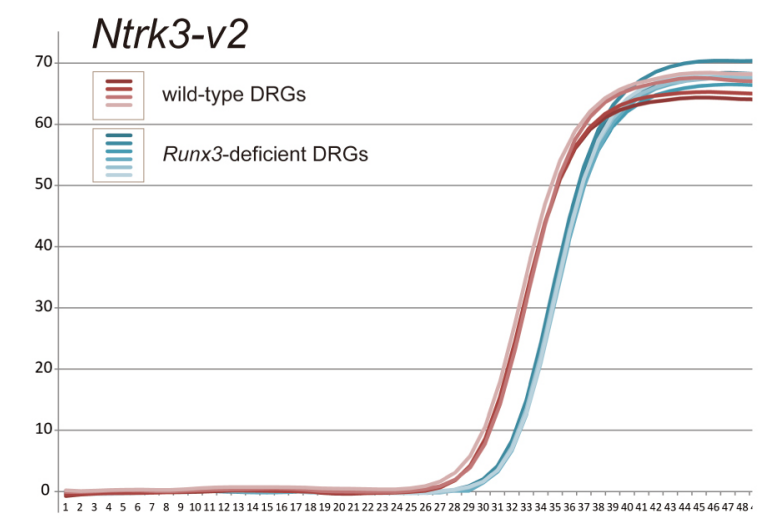

C

- wild-type DRGs

$\square$ Runx3-deficient DRGs

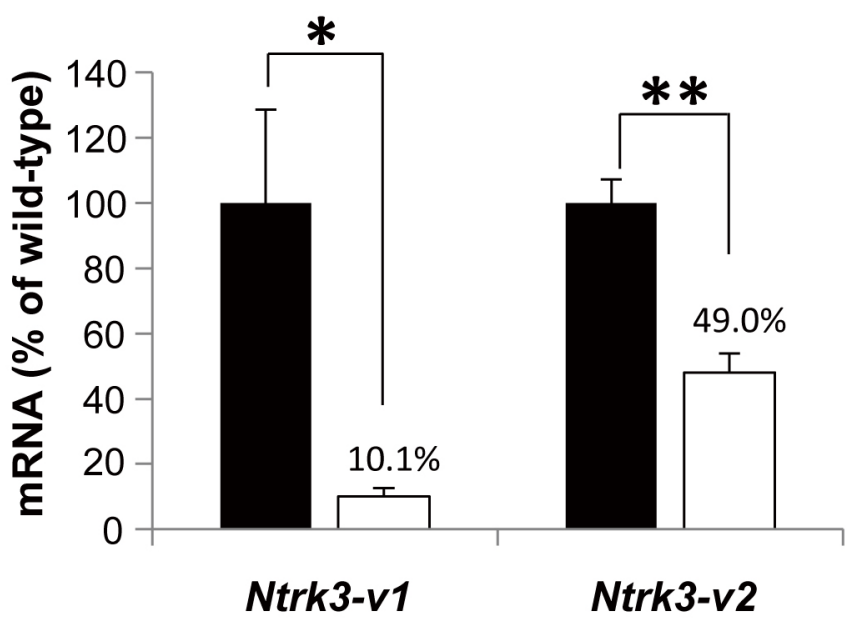

Supporting Information Fig. 2 